\title{
openheart Staged re-evaluation of non-culprit lesions in ST segment elevation myocardial infarction: a retrospective study
}

Troels Thim, Gro Egholm, Kevin Kris Warnakula Olesen, Anne Kaltoft, Christian Juhl Terkelsen, Lars Romer Krusell, Steen Dalby Kristensen, Hans Erik Bøtker, Evald Høj Christiansen, Michael Maeng

To cite: Thim T, Egholm G, Olesen KKW, et al. Staged reevaluation of non-culprit lesions in ST segment elevation myocardial study. Open Heart 2016;3: e000427. doi:10.1136/ openhrt-2016-000427 Revised 18 April 2016 Accepted 3 May 2016 infarction: a retrospective

Received 9 March 2016

\section{ABSTRACT}

Objective: It remains unknown whether complete revascularisation is optimally performed in patients with ST segment elevation myocardial infarction (STEMI) during the index or at staged procedures. The aims of this study were to quantify the number of primary percutaneous coronary intervention $(\mathrm{PCl})$ procedures in which non-culprit lesions needed further evaluation, to determine the consequence of the reevaluation and to quantify adverse cardiac events during the waiting time for re-evaluation and intervention.

Methods: The study was observational and retrospective and included all patients with STEMI treated with primary PCI during 1 year at our centre.

Results: Among the 507 patients with STEMI, 374 were considered sufficiently treated with culprit lesion $\mathrm{PCl}$ only. Complete primary multivessel revascularisation was performed in 11 patients. Nonculprit lesion re-evaluation was planned for 122 patients (24\%). Of these 122 patients, 3 patients died during their index admission. Follow-up data were not available for 3 patients. Among the 116 patients, 187 non-culprit lesions were re-evaluated and 77 patients $(66.4 \%)$ underwent revascularisation with treatment of 119 lesions (63.3\%). Re-evaluation was performed after a median of 30 days (25th centile: 9 days, 75 th centile: 35 days). During the waiting time for reevaluation, two patients underwent a new primary $\mathrm{PCI}$ due to stent thrombosis of the index culprit lesion. Conclusions: Staged re-evaluation of non-culprit lesions observed in patients with STEMI was required in $24 \%$ of a primary PCl cohort. Intervention was performed in $66.4 \%$ of patients scheduled for reevaluation. We observed no adverse events related to the non-culprit lesions during the waiting time for a staged re-evaluation or intervention.

Department of Cardiology, Aarhus University Hospital, Aarhus, Denmark

\section{Correspondence to} Dr Troels Thim; troels.thim@clin.au.dk

\section{INTRODUCTION}

Primary percutaneous coronary intervention (PCI) is the preferred reperfusion strategy

\section{KEY QUESTIONS}

What is already known about this subject?

- Staged complete revascularisation is recommended as the default strategy in ST segment elevation myocardial infarction. Primary complete revascularisation has been suggested as an alternative strategy.

What does this study add?

- This study provides a current estimate of the proportion of patients with ST segment elevation myocardial infarction that are candidates for primary complete revascularisation and the safety of staged complete revascularisation in these patients.

How might this impact on clinical practice?

- Staged complete revascularisation in ST segment elevation myocardial infarction appears to be a safe strategy. Alternative strategies should be evaluated with the current standard as comparator to prove, at least, equal safety.

for patients with ST segment elevation myocardial infarction (STEMI). In patients with STEMI with multivessel disease, guidelines generally recommend treatment of the culprit lesion at the index procedure and staged intervention of non-culprit lesions. ${ }^{1-3}$ New studies have suggested that complete revascularisation should be performed during the initial procedure or during primary admission. ${ }^{4-8}$ Non-culprit lesion PCI during the primary procedure has hitherto predominantly been guided by angiographic assessment of stenoses. ${ }^{6-8}$ Non-culprit lesion severity, however, may be overestimated by angiographic assessment in relation to STEMI. ${ }^{9}$ In elective patients, stenosis evaluation with supplemental methods such as assessment of fractional flow reserve (FFR) may reduce the need for revascularisation 
and improve patient outcomes at the same time. ${ }^{10-13}$ A concern related to complete primary angiographyguided revascularisation is thus an unnecessary treatment of non-significant lesions imposing increased patient risk. $^{45}$

In our centre, we routinely offer staged PCI of presumed significant non-culprit lesions in patients with STEMI with multiple lesions. The aims of the present study were to assess the percentage of primary PCI procedures in which non-culprit lesions needed further evaluation, to determine the consequence of the re-evaluation and to quantify any adverse cardiac events during the waiting time for re-evaluation and intervention.

\section{PATIENTS AND METHODS}

The study is a single-centre observational and retrospective study. According to Danish Law, the study did not require ethical committee approval. The study was approved by the Danish Data Protection Agency (number 2012-41-0164).

Patients treated with primary PCI for STEMI at Aarhus University Hospital from 1 September 2013 to 31 August 2014 were identified in the Western Denmark Heart Registry. ${ }^{14}$ If a patient had more than one primary PCI procedure performed during the study period, only the first primary PCI procedure was included in the study. Patient baseline characteristics and index procedure dates were retrieved from the Western Denmark Heart Registry. ${ }^{14}$

The remaining information was obtained through review of the medical records. We recorded the treatment plan at the index procedure, that is, culprit lesion intervention only, complete primary revascularisation of more than one lesion or staged re-evaluation of nonculprit lesions.

When staged re-evaluation was planned, patients were followed until the time of revascularisation or until a final decision of unneeded revascularisation was taken. The interval between the index primary PCI and revascularisation and adverse cardiac events (death, myocardial infarction or urgent revascularisation) during the waiting time for revascularisation was recorded. For each patient with planned staged re-evaluation, we evaluated whether complete revascularisation at the index primary PCI would have been possible with an acceptable procedure risk and time and contrast use and without the need for heart team consultation. For each lesion scheduled for re-evaluation, we registered the use of additional diagnostic modalities for re-evaluation and the final clinical consequence, that is, coronary bypass surgery, PCI or optimal medical treatment only.

Our strategy for revascularisation in patients with STEMI is in agreement with current guidelines, that is, patients with multivessel disease are offered staged evaluation. $^{1-3}$ On the basis of primary angiogram, re-evaluation is individually tailored to each patient.
Re-evaluation is performed either during the index admission or during a later admission. Re-evaluation may be based on the index coronary angiography alone or supplemented with myocardial perfusion imaging. Re-evaluation may also involve a new coronary angiography with or without supplemental evaluation methods, that is, FFR or intravascular imaging. In any case, heart team consultation may be used either in the evaluation of the index angiography or of subsequent supplemental evaluation. Complex pathologies such as left main lesions, proximal left anterior descending artery lesions or three-vessel disease are, in general, always discussed individually with the heart team. Treatment was, in any case, based on informed consent.

\section{RESULTS}

\section{Patients, adverse events and treatment}

We identified 507 patients undergoing primary PCI during the study period (table 1 ). Of these, 122 patients were scheduled for re-evaluation (figure 1). Follow-up data were not available for three patients who had their re-evaluation performed at other centres (figure 2).

During the follow-up, three patients died before discharge from their index admission. One patient died of sudden cardiac arrest in the coronary care unit 3 days after the index procedure due to myocardial free wall rupture. One patient developed circulatory failure immediately after the index procedure. A myocardial free wall rupture was suspected based on the clinical presentation and echocardiographic findings. This patient was offered acute surgery but refused and died of circulatory collapse. The last patient developed circulatory collapse with pulmonary oedema in the coronary care unit 2 hours after the index procedure.

Table 1 Baseline patient characteristics

Consecutive patients with primary $\mathrm{PCl}$ $(\mathrm{n}=507)$

\begin{tabular}{ll} 
Mean age (SD), years $(n=507)$ & $64.8(12.5)$ \\
Male gender, \% $(n=507)$ & $71.4 \%$ \\
Mean BMI (SD), $\mathrm{kg} / \mathrm{m}^{2}(\mathrm{n}=470)$ & $26.9(4.8)$ \\
Family history of IHD, \% $(n=407)$ & $41.3 \%$ \\
Current smoking, \% $(\mathrm{n}=425)$ & $49.2 \%$ \\
Hypertension, \% $(n=468)$ & $39.1 \%$ \\
Hypercholesterolaemia, \% & $25.4 \%$ \\
$(\mathrm{n}=469)$ & \\
Diabetes, \% $(n=497)$ & $11.4 \%$ \\
Previous AMI, \% $(n=495)$ & $6.1 \%$ \\
Previous PCI, \% $(n=495)$ & $8.3 \%$ \\
Previous CABG, \% $(n=497)$ & $1.2 \%$ \\
\hline
\end{tabular}

Hypertension=treatment with blood pressure lowering agents; hypercholesterolaemia=treatment with lipid lowering agents; diabetes=treatment with antidiabetic drugs or diet.

$\mathrm{AMI}$, acute myocardial infarction; BMI, body mass index; CABG, coronary artery bypass grafting; IHD, ischaemic heart disease; $\mathrm{PCl}$, percutaneous coronary intervention. 


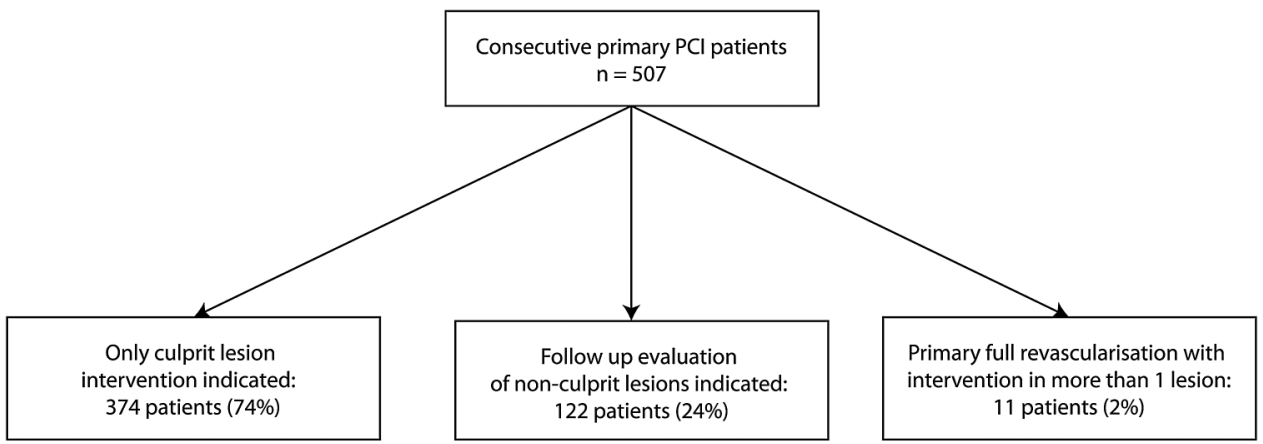

Figure 1 Patient flow chart. $\mathrm{PCl}$, percutaneous coronary intervention.

Echocardiography confirmed papillary muscle rupture. Acute surgery was performed but the patient died of multiple organ failure in the intensive care unit 3 days after surgery.

During the follow-up, two patients suffered from acute myocardial infarction and underwent urgent PCI. Stent thrombosis of the index lesion was the cause of recurrent myocardial infarction in both these patients. After this second primary PCI procedure, both patients had elective re-evaluation as originally planned.

We did not identify any adverse events caused by the non-culprit lesions in the patients scheduled for staged re-evaluation.

Among the 122 patients scheduled for re-evaluation, the treating PCI operator retrospectively considered that full primary revascularisation would have been feasible in 62 patients (51\%) with an acceptable procedure risk and time and contrast use and without the need for heart team consultation. Reasons for considering primary full revascularisation infeasible included the need for extensive revascularisation, complex lesion morphology including chronic total occlusions and the need for heart team consultation.

Treatment based on re-evaluation is reported in figure 2.

\section{Re-evaluation modalities}

Among the 116 patients with follow-up evaluation, 100 patients had a new coronary angiogram as part of their evaluation (figure 3). These 100 patients had a total of 147 non-culprit lesions. This angiographic analysis was supplemented with evaluation using FFR (41 patients with 56 lesions), instantaneous wave-free ratio (3 patients with 4 lesions), intravascular ultrasound ( 2 patients with 2 lesions) and positron emission tomography (1 patient with 1 lesion). Subsequent heart team consultation was used in the evaluation of 21 patients with 37 lesions. Among these 21 patients, 9 were treated with bypass surgery, 8 were treated with PCI, while 4 received optimal medical therapy and no further revascularisation. The remaining 79 patients with 110 lesions were treated without heart team consultation based on the new coronary angiogram supplemented with FFR (36

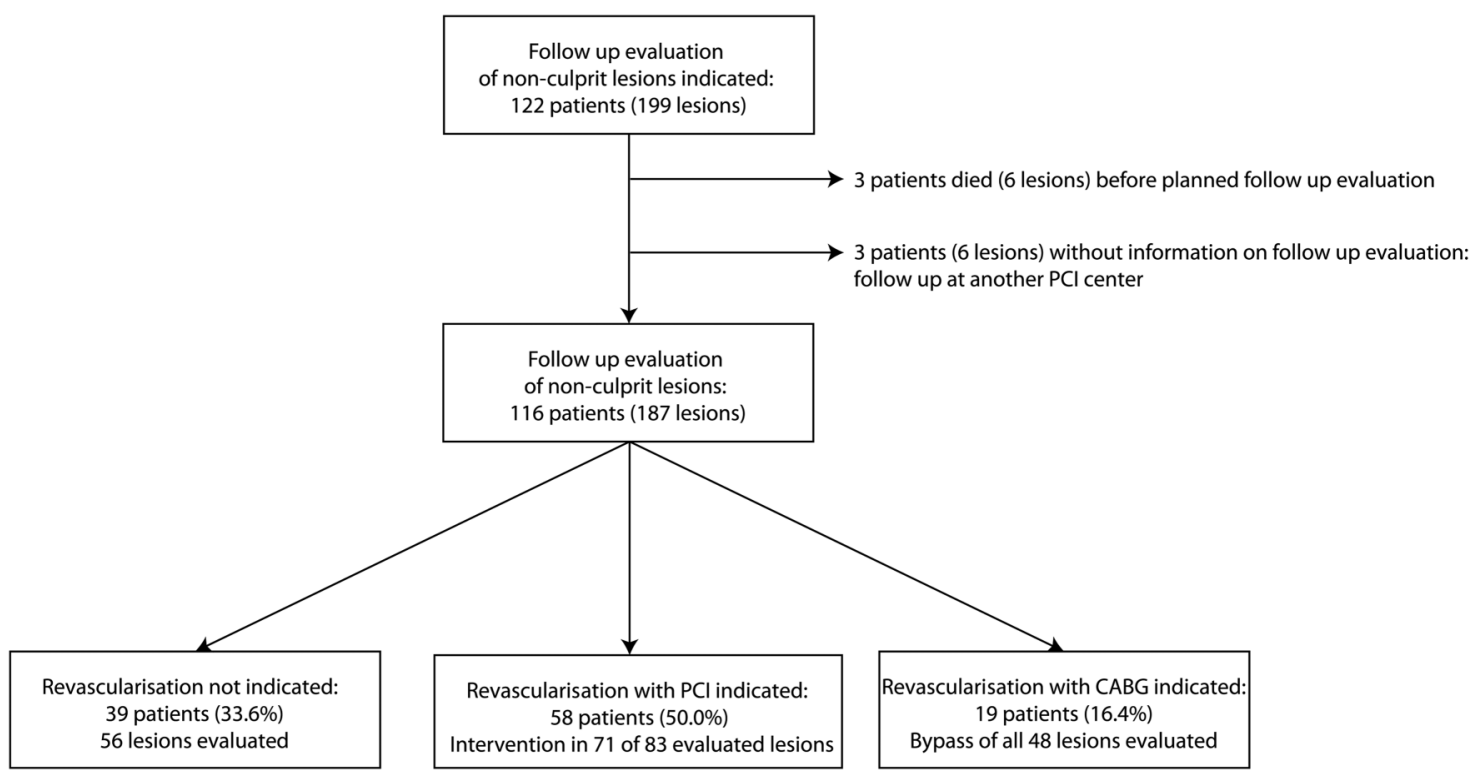

Figure 2 Follow-up evaluation results of non-culprit lesions. CABG, coronary artery bypass grafting; PCl, percutaneous coronary intervention. 


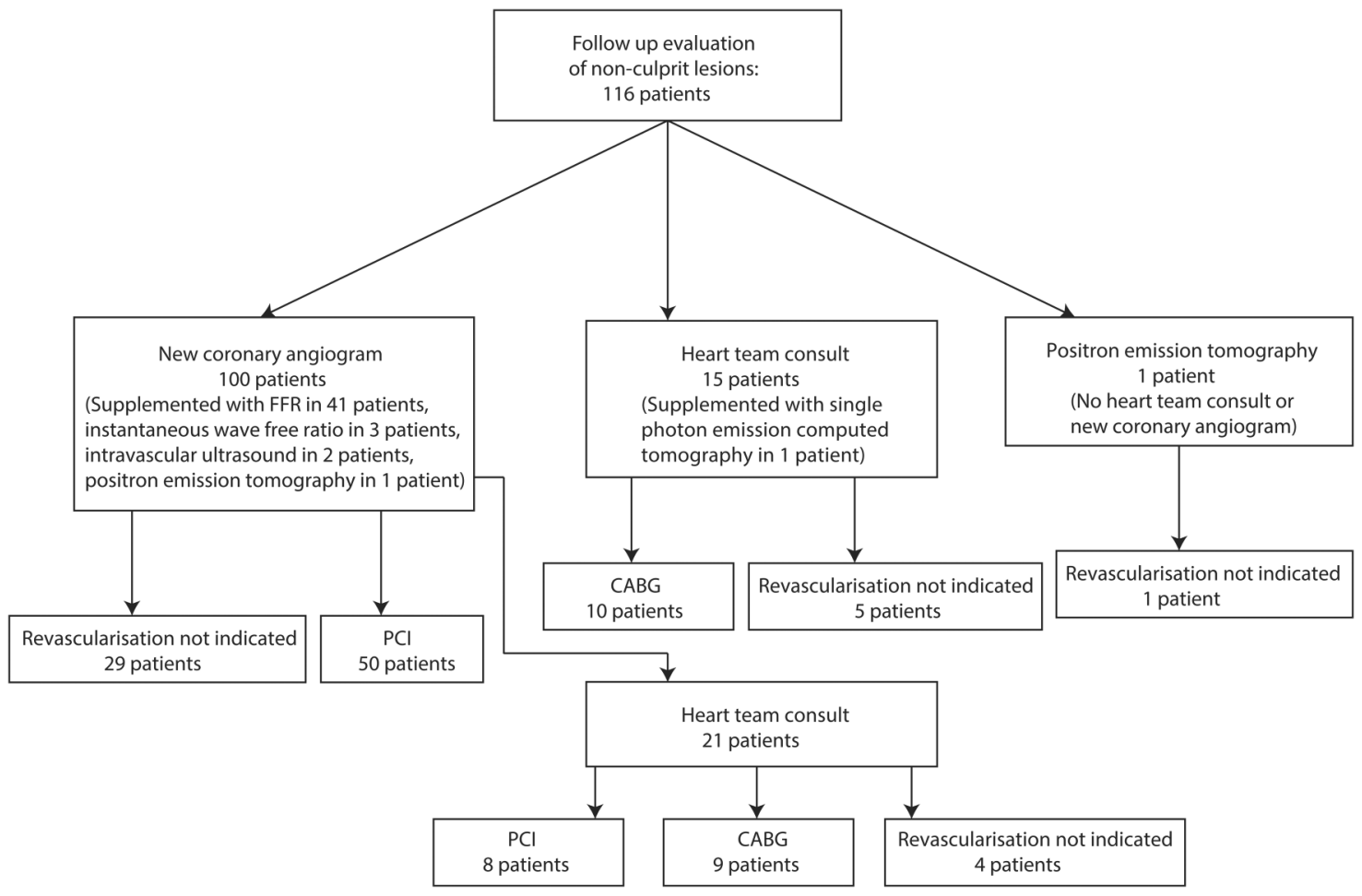

Figure 3 Re-evaluation modalities. CABG, coronary artery bypass grafting; FFR, fractional flow reserve; PCl, percutaneous coronary intervention.

patients), instantaneous wave-free ratio (3 patients) and intravascular ultrasound ( 2 patients). Of these, 50 patients were treated with PCI while 29 received optimal medical therapy and no further revascularisation.

Among the remaining 16 patients with 40 lesions, re-evaluation was based on baseline coronary angiogram supplemented with single-photon emission CT and positron emission tomography in two patients. Heart team consultation was used in the evaluation of 15 of these 16 patients. Among these 15 patients, 10 were treated with bypass surgery and 5 received optimal medical therapy and no further revascularisation. The remaining patient received optimal medical therapy and no further revascularisation at the discretion of the cardiologist based on negative myocardial perfusion imaging.

\section{Timing of re-evaluation}

Follow-up evaluation ( $\mathrm{n}=116$ patients) was performed at a median of 30 days after the index procedure (25th centile: 9 days, 75 th centile: 35 days). Follow-up during the index admission $(n=29)$ was performed at a median of 1 day after the index procedure (25th centile: 1 day, 75th centile: 4 days). In these 29 patients, treatment decision was based on new coronary angiography without heart team consultation (9 patients), with heart team consultation based on index angiography (14 patients) or based on new coronary angiography followed by heart team consultation (6 patients). Follow-up after discharge from the index admission $(n=87)$ was performed at a median of 32 days after the index procedure (25th centile: 28 days, 75 th centile: 37 days). Among these 87 patients, 85 had a new coronary angiography and 2 were evaluated with myocardial perfusion scans.

Patients scheduled for bypass surgery $(n=19)$ experienced an additional differentiated waiting time before final revascularisation with a median 45 days after the evaluation date (range 5-96 days). Five patients underwent coronary artery bypass grafting during the index admission with a median waiting time of 7 days after re-evaluation (range 5-7 days). Among the remaining 14 patients scheduled for bypass surgery after discharge from the index admission, bypass surgery was performed at a median of 60 days after re-evaluation (range 1296 days). We observed no adverse cardiac events during the interval from follow-up evaluation to bypass surgery. The bypass surgery procedures were either minimally invasive or open procedures.

\section{DISCUSSION}

In this cohort of patients with STEMI, $24 \%$ were scheduled for re-evaluation of non-culprit lesions. Among these, $66 \%$ needed further revascularisation. Re-evaluation was individually tailored regarding methods and timing of re-evaluation. On the basis of individual assessment, re-evaluation was performed either during the index admission or after discharge. None of the non-culprit lesions caused events in the waiting time for re-evaluation. Staged re-evaluation thus appears to be a safe approach. 


\section{Complete revascularisation}

In principle, three different revascularisation strategies for patients with STEMI with multivessel disease exist, that is, culprit lesion primary PCI only, primary complete revascularisation with PCI or staged complete revascularisation. Regarding culprit lesion intervention only four randomised clinical trials have indicated that this strategy is inferior to complete revascularisation. ${ }^{6-8}{ }^{15}$ Thus, the current data favour complete revascularisation as already reflected in the guidelines. ${ }^{1-3}$

The questions that remain to be answered is the optimal timing and the optimal methodology to guide complete revascularisation, that is, angiography guided at the index procedure, ${ }^{6}$ guided by functional assessment (eg, FFR) at the index procedure or during the index admission, ${ }^{7}$ guided by functional assessment before discharge from index admission ${ }^{15}$ or during a subsequent admission guided by myocardial perfusion imaging, pressure wire measurements or angiography only.

Our study adds important information to the randomised studies. First, in our real-life data on consecutive patients with STEMI and an acute angiogram showing significant multivessel disease, approximately one-third of patients did not need further revascularisation. If the decision to revascularise non-culprit lesions were based on angiographic assessment at the index procedure only, it seems evident that unnecessary revascularisation could be performed in some of these patients. ${ }^{11-13}$ Furthermore, it has been shown that non-culprit lesion severity can be overestimated in patients with STEMI at the index procedure, ${ }^{9}$ which may in turn partly explain why acute multivessel PCI has been associated with increased mortality when compared with staged PCI of non-culprit lesions. ${ }^{4516}$ A second, staged, intervention, even with a median waiting time of 30 days for re-evaluation, appears safe with no events related to nonculprit lesions during the waiting time. Third, approximately half of the patients with STEMI with multivessel disease had lesions that the treating PCI operator did not find feasible for complete primary revascularisation. Although this evaluation was subjective, these patients are unlikely to have been included in randomised studies comparing complete primary versus complete staged revascularisation, and our study thus adds quantitative data on the external validity of these previous randomised clinical trials. ${ }^{6-8}$

\section{Supplemental lesion evaluation}

In our study, $34 \%$ of patients with STEMI with multivessel disease and staged re-evaluation of non-culprit lesions did not have significant lesions at re-evaluation. Staged re-evaluation was mainly guided by angiography alone, FFR or instantaneous wave-free ratio measurements. Our data, combined with data showing that angiography exaggerates non-culprit lesion severity at the index procedure in patients with STEMI, ${ }^{9}$ suggest that additional functional assessment is frequently necessary to distinguish the significant from the non-significant non-culprit lesions. The approach is well established in stable coronary artery disease. ${ }^{11}{ }^{12}$ Although acute functional evaluation of non-culprit lesions by FFR is feasible in patients with STEMI, ${ }^{13}$ the approach has not become standard. FFR, however, has been used in the staged evaluation of non-culprit lesions before discharge. ${ }^{15}$ As compared with FFR, evaluation by instantaneous wavefree ratio is a simpler and faster method to evaluate lesion severity, ${ }^{17-19}$ but so far no study has validated its usefulness for the assessment of non-culprit lesions in patients with STEMI during the index procedure. Finally, non-invasive myocardial perfusion imaging methods can also be used to evaluate the clinical significance of non-culprit lesions.

\section{Clinical implications}

This study provides real-life data on the management of non-culprit lesions detected in relation to acute angiography for STEMI. Our data support that staged evaluation in accordance with current guideline recommendations is safe, even with a median time to re-evaluation of 30 days. The approach allows careful decision-making, heart team evaluation and optimal planning of the revascularisation strategy. With no events related to non-culprit lesions in this study, a significant improvement in safety by complete primary revascularisation may be difficult to obtain, even with supplemental evaluation methods used during the primary procedure. However, complete primary revascularisation may be justified, at least in selected patients, since patient risk and system costs related to an additional staged procedure may be reduced. Patient selection for complete primary revascularisation, staged revascularisation or clinical follow-up only remains a subject for further research. In particular, the role of supplemental diagnostic modalities such as intracoronary pressure measurements at the primary PCI procedure awaits further investigation.

Culprit lesion intervention only, in the presence of one or more other significant lesions, may be considered an inappropriate control treatment in future studies since it appears inferior to complete revascularisation. ${ }^{4-7} 15$ Our data suggest that staged full revascularisation is safe and, consequently, should be considered as the relevant control treatment for future randomised clinical trials focusing on complete primary revascularisation in STEMI.

\section{Limitations}

Our study is a single-centre observational study and provides no information on the differences between strategies or on the optimal timing of complete revascularisation. Nevertheless, our data strongly support that staged evaluation, as recommended by guidelines, is a very safe approach.

Three-quarters of the patients had culprit lesion intervention only. Although the majority had single-vessel disease, a number of these patients had lesions other 
than the culprit lesions. It must be expected that the operators in these cases used their clinical judgement to waive further evaluation of multivessel disease based on relevant comorbidity or suspected clinical insignificance of chronic total occlusions or lesions in minor side branches. We did not evaluate such patients since further revascularisation was considered undemanding by the PCI operator.

\section{CONCLUSIONS}

Staged re-evaluation of non-culprit lesions observed in patients with STEMI was required in $24 \%$ of a primary PCI cohort. Intervention was performed in $66 \%$ of patients scheduled for re-evaluation. We observed no adverse events related to the non-culprit lesions during the interval for a staged re-evaluation or intervention. The strategy of staged re-evaluation of non-culprit lesions after STEMI appears to be safe.

Contributors Study planning was done by TT, GE, KKWO, SDK, HEB, EHC and MM. Data collection was carried out by TT, AK, CJT, LRK, SDK, HEB, EHC and MM. Data analysis and interpretation were carried out by TT, GE, KKWO, AK, CJT, LRK, SDK, HEB, EHC and MM. TT drafted the manuscript, and along with GE, KKWO, AK, CJT, LRK, SDK, HEB, EHC and MM made a critical revision of it. TT, GE, KKWO, AK, CJT, LRK, SDK, HEB, EHC and MM approved the final manuscript for publication.

Competing interests None declared.

Ethics approval Head of Department of Cardiology, Aarhus University Hospital.

Provenance and peer review Not commissioned; externally peer reviewed.

Data sharing statement No additional data are available.

Open Access This is an Open Access article distributed in accordance with the Creative Commons Attribution Non Commercial (CC BY-NC 4.0) license, which permits others to distribute, remix, adapt, build upon this work noncommercially, and license their derivative works on different terms, provided the original work is properly cited and the use is non-commercial. See: http:// creativecommons.org/licenses/by-nc/4.0/

\section{REFERENCES}

1. Steg PG, James SK, Atar D, et al. Task Force on the management of ST-segment elevation acute myocardial infarction of the European Society of Cardiology (ESC). ESC guidelines for the management of acute myocardial infarction in patients presenting with ST-segment elevation. Eur Heart J 2012;33:2569-619.

2. O'Gara PT, Kushner FG, Ascheim DD, et al. 2013 ACCF/AHA guideline for the management of ST-elevation myocardial infarction: a report of the American College of Cardiology Foundation/American Heart Association Task Force on Practice Guidelines. Circulation 2013;127:e362-425.

3. Windecker S, Kolh P, Alfonso F, et al. Authors/Task Force Members. 2014 ESC/EACTS Guidelines on myocardial revascularization: the Task Force on Myocardial Revascularization of the European Society of Cardiology (ESC) and the European Association for Cardio-Thoracic Surgery (EACTS) developed with the special contribution of the European Association of Percutaneous Cardiovascular Interventions (EAPCI). Eur Heart $J$ 2014;35:2541-619.

4. Vlaar PJ, Mahmoud KD, Holmes DR Jr, et al. Culprit vessel only versus multivessel and staged percutaneous coronary intervention for multivessel disease in patients presenting with ST-segment elevation myocardial infarction: a pairwise and network meta-analysis. J Am Coll Cardiol 2011;58:692-703.

5. Bainey KR, Mehta SR, Lai T, et al. Complete vs culprit-only revascularization for patients with multivessel disease undergoing primary percutaneous coronary intervention for ST-segment elevation myocardial infarction: a systematic review and meta-analysis. Am Heart J 2014;167:1-14.e2.

6. Wald DS, Morris JK, Wald NJ, et al. Randomized trial of preventive angioplasty in myocardial infarction. $N$ Engl $\mathrm{J} \mathrm{Med}$ 2013;369:1115-23.

7. Gershlick AH, Khan JN, Kelly DJ, et al. Randomized trial of complete versus lesion-only revascularization in patients undergoing primary percutaneous coronary intervention for STEMI and multivessel disease: the CvLPRIT trial. J Am Coll Cardiol 2015;65:963-72.

8. Politi L, Sgura F, Rossi R, et al. A randomised trial of target-vessel versus multi-vessel revascularisation in ST-elevation myocardial infarction: major adverse cardiac events during long-term follow-up. Heart 2010;96:662-7.

9. Hanratty CG, Koyama $\mathrm{Y}$, Rasmussen $\mathrm{HH}$, et al. Exaggeration of nonculprit stenosis severity during acute myocardial infarction: Implications for immediate multivessel revascularization. J Am Coll Cardiol 2002;40:911-16.

10. Tonino PA, De Bruyne B, Pijls NH, et al. Fractional flow reserve versus angiography for guiding percutaneous coronary intervention. N Engl J Med 2009;360:213-24.

11. De Bruyne B, Pijls NH, Kalesan B, et al. Fractional flow reserve-guided $\mathrm{PCl}$ versus medical therapy in stable coronary disease. N Engl J Med 2012;367:991-1001.

12. De Bruyne B, Fearon WF, Pijls NH, et al. Fractional flow reserve-guided $\mathrm{PCl}$ for stable coronary artery disease. N Engl J Med 2014;371:1208-17.

13. Ntalianis A, Sels JW, Davidavicius G, et al. Fractional flow reserve for the assessment of nonculprit coronary artery stenoses in patients with acute myocardial infarction. JACC Cardiovasc Interv 2010;3:1274-81.

14. Schmidt M, Maeng M, Jakobsen CJ, et al. Existing data sources for clinical epidemiology: the Western Denmark Heart Registry. Clin Epidemiol 2010;2:137-44.

15. Engstrom $\mathrm{T}$, Kelbaek $\mathrm{H}$, Helqvist $\mathrm{S}$, et al. Complete revascularisation versus treatment of the culprit lesion only in patients with ST-segment elevation myocardial infarction and multivessel disease (DANAMI-3-PRIMULTI): an open-label, randomised controlled trial. Lancet 2015;386:665-71.

16. Jensen LO, Thayssen P, Farkas DK, et al. Culprit only or multivessel percutaneous coronary interventions in patients with ST-segment elevation myocardial infarction and multivessel disease. Eurolntervention 2012;8:456-64.

17. Sen S, Escaned J, Malik IS, et al. Development and validation of a new adenosine-independent index of stenosis severity from coronary wave-intensity analysis: results of the ADVISE (ADenosine Vasodilator Independent Stenosis Evaluation) study. J Am Coll Cardiol 2012;59:1392-402.

18. Sen S, Asrress KN, Nijjer S, et al. Diagnostic classification of the instantaneous wave-free ratio is equivalent to fractional flow reserve and is not improved with adenosine administration. Results of CLARIFY (Classification Accuracy of Pressure-Only Ratios Against Indices Using Flow Study). J Am Coll Cardiol 2013;61:1409-20

19. Petraco R, Escaned J, Sen S, et al. Classification performance of instantaneous wave-free ratio (iFR) and fractional flow reserve in a clinical population of intermediate coronary stenoses: results of the ADVISE registry. Eurolntervention 2013;9:91-101. 